\title{
A liquid level control system based on LabVIEW and MATLAB hybrid programming
}

\author{
Zhen $\mathrm{Li}^{\dagger}$, Ping Ma and Wei Liang, \\ Department of automation, North China Electric Power University, \\ Baoding, 071003, China \\ ${ }^{\dagger}$ Email:18822370603@163.com \\ www.ncepu.edu
}

\begin{abstract}
This control system is designed for process control experiment platform, which combines the advantages of LabVIEW and MATLAB , avoid the shortcomings of the two. Double tank water level control is the object of the system, using PCI data acquisition card to collect the data and send control signals, based on LabVIEW and MATLAB mixed programming to develop the control system. Solved the shortcomings of the poor ability to calculate the matrix of LabVIEW, and also solved the problem of MATLAB in the data communication. The advantages of efficient communication between PCI data acquisition card and LabVIEW are used in the system, and the powerful computing power of MATLAB is also used in the systerm. In the case of limited laboratory resources, the control system has good experiment and teaching effect.
\end{abstract}

Key words: LabVIEW and MATLAB hybrid programming; liquid level control; experimental platform; virtual instrument.

\section{Introduction}

In the production process, the process control of the field situation is complicated and changeable, so the actual situation and the theory will often have a certain gap [1]. Therefore, in the teaching experiment, we need to try to simulate the situation of the field, but because of the limitation of the experimental equipment, most of the process control experiments are based on the software simulation. Learners lack of understanding for the object characteristics ,performance of the controller, actuator action in solving practical problems, so in the face of the actual problem of lack of experience.

By using LabVIEW to build virtual instrument with the development of high efficiency, strong maintainability, testing accuracy, stability and reliability; saving investment, easy equipment renewal and function expansion, but did not have any advantages in most matrix calculation and control system of the algorithm, which limits the application of LabVIEW [2-3]. MATLAB has a scientific calculation and powerful matrix calculation function, can provide a large and reliable algorithm, numerical analysis, matrix computation, signal 
processing in one, according to the different areas have special toolbox[4-5].But MATLAB in the user interface development ability is poor. MATLAB In data communication, hardware control is more complicated. This limits the scope of application of MATLAB[6-7].

So the control system based on the actual physical experiment platform has a very important significance in teaching. The comprehensive advantages of LabVIEW and MATLAB, to avoid their disadvantages, give full play to the advantages of LabVIEW in interface design, data communication, hardware control, and exert the powerful calculation function of MATLAB, developed the process control system based on mixed programming of LabVIEW and MATLAB.

\section{System Block Diagram}

In this paper, we will introduce the system by use as an example to control the water level of double tank, because the double tank level control after adding delay can simulate the control of most industrial processes. The structure of this design system is shown below.

Fig. 1. General block diagram of the system

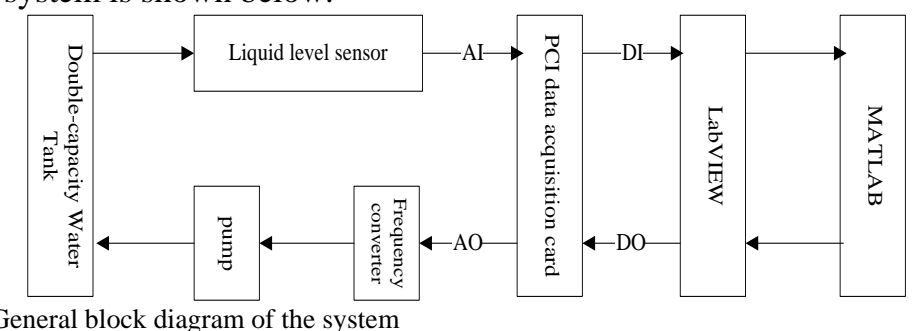

The liquid level transmitter is a pressure sensor mounted on the bottom of the water tank, and the output current signal $(4-20 \mathrm{~mA})$ is converted by the acquisition card. Acquisition card and PC side using serial communication, because the acquisition card using the RS485 protocol, the use of RS232 computer protocol, it is necessary to convert the 485 level to 232 level[8]. After the data is processed by LabVIEW, the algorithm is calculated by the MATLAB calculation and the result is returned to the LabVIEW. The current signal of the 4-20mA signal of the acquisition card is made to adjust the speed of the water pump motor to reach the goal of controlling the water level of the water tank[9].

\section{Design of Control System}

\subsection{Main VI}

In LabVIEW programming, the main loop of the program is to use the While loop structure, in this loop structure, the sequence of three frames is nested, each 
frame sequence structure is nested according to different functional requirements, such as the event structure, the While loop structure, the if loop structure, and so on. As shown below, the flow chart of the main program structure.

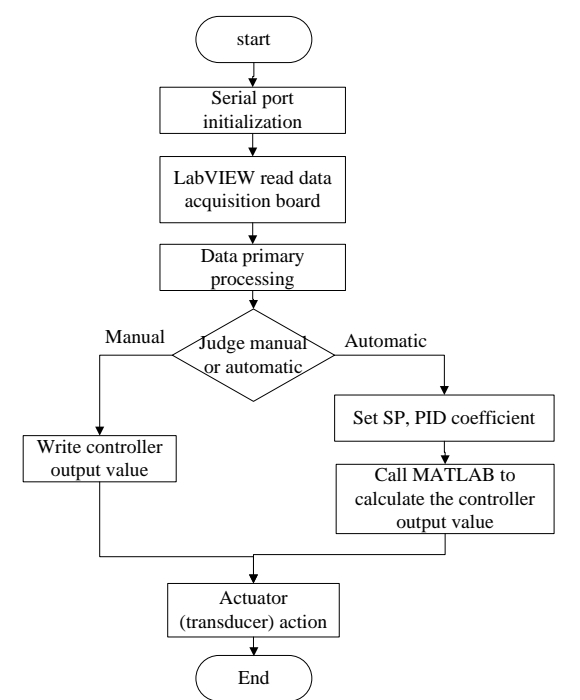

Fig. 2. Flow chart of the main VI program

The main interface as shown at the fifth chapter of the thesis, the interface is roughly divided into two parts. Left interface, including the waveform display interface, the resources of the serial port selection button, save the data button. The right part of the interface includes a double tank model simulation, display real-time status of the actual physical model experiment observation operator convenience. Before the experiment, the error of the liquid level measurement can be corrected by the level correction button, so that the experiment has a better control effect, Click the "Frequency converter" button to pop up the status of the configuration dialog box, configure the manual or automatic state of motion as well as manual output values, PID parameters, the target value of the liquid level and other parameters.

\subsection{LabVIEW and MATLAB mixed programming}

LabVIEW and MATLAB mixed programming has four ways: (1) based on Activex and MATLAB Server for communication (2) COM component method (3) intermediate file transfer method (4) based on dynamic link library (DLL) method [10].

This design in the comparison of the advantages and disadvantages of the above methods, the premise, according to the need to choose the first method Script 
MATLAB node. It is based on the communication between Activex and Server MATLAB to complete the data exchange. MATLAB Script node with multiple input, multiple output characteristics, each call can handle a large amount of information. MATLAB script can be debugged in MATLAB, and then imported into the MATLABSeript node. MATLAB Script node for the input and output data types have a clear requirement, only the data type of LabVIEW and MATLAB in the data type matching, in order to carry out data transmission. Using MATLAB Script node is quick and convenient, but it is not conducive to the development of large application. When it is needed, it can be modularized, and the main program can be used for dynamic loading.

\subsection{Configuration dialog}

In order to save the main interface space, in order to make the main interface more concise and more humane, the configuration and the design of parameters of the system are used in the form of the dialog box. Click the control valve button or the transducer button to pop up the dialog box to set the system status and parameters. In the pop-up dialog box, you can configure the system manual or automatic switching, manual output of a given value, set value, PID parameters. Can also be real-time monitoring of the controller output, real-time comparison between the given value and the measured value of the gap.

Fig. 3. State configuration dialog

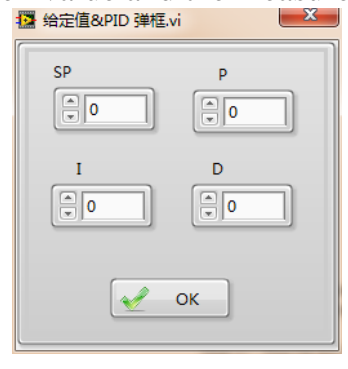

When you click on the "inverter" button on the main interface, the dialog box is shown in the Fig. 4(left),it mainly consists of three parts:(1)The upper part of the dialog box shows the given value and the real value;(2)The middle of the dialog box shows the status of the controller in real time;(3) The lower part of the dialog box is used to switch between the manual state and the automatic state, you can set the controller output value configuration in the manual state, click the "Settings" button in the automatic state will pop up the dialog box above, which can be easily configured to automatically control the given values and PID parameters. 


\subsection{Sub-VI}

This design uses the serial communication method to carry on the data reading and writing the control instruction, LabVIEW comes with the VISA serial function is used in the serial port configuration and serial read and write

In the design of hardware interface, the Modbus communication protocol is used between LabVIEW and PCI acquisition card. When using the Modbus communication protocol, data transmission must follow certain rules, so the communication data frame generation is a sub VI program called multiple times. 06 single register read code and 03 holding registers preset code were used.

CRC checksum is a cyclic redundancy check, different data have different check codes, the recipient will check the code in order to confirm that the transfer is correct. The verification code generation sub VI program is designed to calculate the CRC code for each group of data. Since the calculation of the $\mathrm{CRC}$ code in the process involves a lot of data calculation, so call the MATLAB server to calculate.

\section{Application Examples}

In order to establish the model of the double tank water, the step response curve of the system is obtained when the system is in manual state. After many experiments, we found that the level of the top water tank and the below water tank is suitable for when the output of the controller is $50 \%$. At this time, the level of the top water tank and the below water tank are $10 \mathrm{~cm}$ and $5 \mathrm{~cm}$. Giving a $5 \%$ step signal to the system, the stable level are about $15 \mathrm{~cm}$ and $10 \mathrm{~cm}$. The following image(left) is the system step response curve.

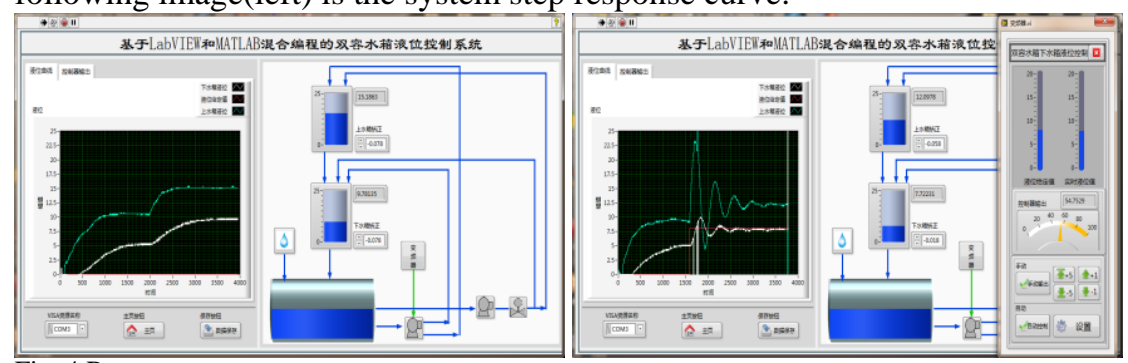

Fig. 4.Response curve

Establishing the model of this system after obtaining the step response curve. Tuning the regulator parameters using the stability boundary method or the attenuation curve method. The above picture(right) is the response curve under automatic control. 


\section{Conclusion}

This design successfully applies the technology of LabVIEW and MATLAB mixed programming in the design of control system. This paper applies the design to the actual control system and evaluates it. The results of this experiment is satisfactory. Practice process proves that, the technology mixed LabVIEW and MATLAB combines the advantages of the two software and avoids the disadvantages. The system not only has high efficient computing power and accurate data communication, but also has a good man-machine interface. This design uses the MATLAB Script node to carry on the algorithm computation, at the same time has the modular programming style. So it has better portability and more extensive use of space. This design is not only limited to the PID control algorithm and the single loop control system, but also can use a more advanced control algorithm.(such as: Predictive Control, Adaptive Control, Fuzzy Control, etc).

\section{References}

1. Y. H. Jin., Process Control,( Tsinghua University Press, Beijing,1993).

2. X. J. Wang, Serial port sampling data processing using LabVIEW, Electronic Measurement Technology ,Vol.37 No.3(2014),pp.108-110.

3. F. Xu and M. L. He (eds), The Mixed Programming of LabVIEW and Matlab Based on Database and Its Realization, Measurement and Control Technology,Vol26 No.2(2007), pp.70-72.

4. L. K. Fang, Process Control and Its Realization by MATLAB,( Electronic Industry Press,Beijing,2013).

5. W. D. Liu and D. W. Wang, MATLAB Simulation of Process Control System, ( Machinery Industry Press, Beijing,2009).

6. X. J. Fu, Process computer control system,( Northwestern Polytechnical University Press, Xian,1995).

7. S. F. Yao and D. Q. Xue (eds), Mixed Programming of LabVIEW and MATLAB, Software Technology,Vol.24 No.6(2005),pp.111-112.

8. Y. L. Wang and Z. H. Xiong (eds), Seamless Integration of LabVIEW and MATLAB, Computer Application,Vol.26 No.3(2006),pp.659-698.

9. Robert H. Bishop , LabVIEW Practice Tutorial,( Electronic Industry Press, Beijing, 2014).

10. Y. N. Guo and J. J. Chang (eds), Process control system,( Machinery Industry Press,Beijing,2009). 